\section{CEACLIN, un instrumento en español para identificar estrategias para el aprendizaje de la clínica de estudiantes de medicina. Desarrollo y validación}

\author{
MARCELA BITRAN ${ }^{1, \mathrm{a}}$, OSLANDO PADILLA ${ }^{2, \mathrm{~b}}$, DENISSE ZÚÑIGA ${ }^{1, \mathrm{c}}$, \\ ISABEL LEIVA $^{1,3}$, MARIBEL CALDERÓN ${ }^{4, d}$, ARNOLDO RIQUELME ${ }^{1,5}$
}

\section{CEACLIN, an instrument suited to identify medical students' strategies to learn in pre-clerkship years}

Background: Upon the beginning of pre-clerkship years, medical students must develop strategies to learn from experience and to improve their relational skills to communicate with patients. Aim: To develop an instrument to identify the strategies used by medical students to learn in clinical contexts. Material and Methods: Using a Delfi technique to reach consensus, a national panel of students and clinical teachers from 15 Chilean medical schools analyzed an 80item questionnaire built from perceptions of Chilean students and teachers from one medical school. After two Delfi rounds and a pilot application, a 48-item questionnaire was obtained. Its reliability and construct validity were assessed by Cronbach alpha coefficient and factor analysis, respectively, on the base of an application to 336 medical students. Results: The questionnaire developed, named CEACLIN, is highly reliable $(\alpha=0.84)$. Its inner structure is made of eleven factors: Autonomy, Solving doubts and problems, Searching and organizing information, Proactivity, Reaching to others, Paying attention and emotions, Searching for trust, Evading burden, Coping with burden, Motivation and Postponing the personal life. All together, these factors account for $47.4 \%$ of the variance. Conclusions: CEACLIN is a valid, reliable and easy to use instrument suited to identify students' strategies to learn in pre-clerkship years. Many of its items allude to concepts of theories of experiential learning and motivation. We hope that CEACLIN will be of value to medical students and clinical teachers to improve the learning and teaching of clinical reasoning and communication skills.

(Rev Med Chile 2015; 143: 1295-1305)

Key words: Clinical clerkship; Problem based learning; Questionnaires; Students, medical; Test taking skills.
${ }^{1}$ Centro de Educación Médica, Escuela de Medicina, Pontificia Universidad Católica de Chile. 2Departamento de Salud Pública, Escuela de Medicina, Pontificia Universidad Católica de Chile. ${ }^{3}$ Departamento de Enfermedades Respiratorias, Escuela de

Medicina, Pontificia Universidad Católica de Chile.

${ }^{4}$ Escuela de Psicología, Pontificia Universidad Católica de Chile.

${ }^{5}$ Departamento de

Gastroenterología, Escuela de

Medicina, Pontificia Universidad

Católica de Chile.

aBioquímica, PhD en

Farmacología.

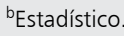

'Psicóloga, Magíster en

Psicología.

${ }^{d}$ Magíster en Psicología, PhD@ en Psicología.

Investigación financiada por el Fondo Nacional de Desarrollo Científico y Tecnológico FONDECYT, proyectos \# 1120534 (M.B.) y \# 1120652 (A.R.)

Recibido el 12 de marzo de 2015 aceptado el 15 de julio de 2015 .

Correspondencia a:

Marcela Bitran

Centro de Educación Médica, Escuela de Medicina, Pontificia Universidad Católica de Chile. Fono: 562-23543811 mbitran@med.puc.cl
$\mathrm{D}$ urante el período comprendido entre los primeros años de la carrera, y el internado, la forma de enseñar y aprender la medicina cambia radicalmente ${ }^{1-3}$. La asistencia a clases y el estudio individual dan paso a actividades prácticas individuales y grupales, primero en situaciones simuladas y luego en escenarios reales, hospitalarios o ambulatorios. A partir de tercer o cuarto año, los estudiantes se dedican fundamentalmente a aprender a realizar la entrevista clínica y el examen físico, y a integrar sus conocimientos previos con estas experiencias prácticas ${ }^{1-3}$.

En estos años, los estudiantes experimentan emociones encontradas y crecientes niveles de 
ansiedad y estrés ${ }^{4-6}$. Aunque les entusiasma interactuar y aprender con pacientes reales, muchos no saben bien cómo proceder en estos escenarios prácticos donde las estrategias que usaron en los primeros años de estudio no parecen servir ${ }^{4,7,8}$.

Es que, en efecto, la facilidad para aprender en situaciones prácticas que involucren el contacto con otras personas no es una característica frecuente de los estudiantes de medicina. En nuestro país, la mayoría destaca por lo contrario: su capacidad de asimilar pasivamente gran cantidad de información y reflexionar sobre ella en forma individual ${ }^{9,10}$. Este perfil, llamado Asimilador ${ }^{11,12}$, es funcional para los años iniciales de formación, pero no así para el ciclo preclínico y clínico, cuando son indispensables la proactividad, autonomía y las habilidades relacionales y de trabajo en equipo ${ }^{4,7,13}$.

Sin embargo, contrariamente a lo sostenido por décadas, los estilos de aprendizaje pueden cambiar ${ }^{14-16}$. Este fenómeno lo observamos durante el seguimiento a dos cohortes de estudiantes ${ }^{16}$ : Una gran proporción de los estudiantes que, al ingresar a medicina, se definió como Asimilador; se identificó -al final de la carrera- como Convergente $^{11}$ : un aprendiz activo, motivado por resolver problemas concretos. Este estudio reveló la capacidad de los estudiantes para adaptarse a los cambios curriculares; sin embargo, no permite comprender el proceso que subyace a esta transformación.

Dada la falta de instrumentos para evaluar estrategias para el aprendizaje clínico, algunos investigadores han adaptado cuestionarios creados para el aprendizaje en salas de clases, como el Study Process Questionnaire, y lo han usado para conocer las aproximaciones de los estudiantes de medicina de Indonesia al aprendizaje clínico ${ }^{17}$. Ésta nos parece una estrategia arriesgada en términos de la validez, dadas las diferencias cualitativas entre el aprendizaje teórico y el experiencial.

Para resolver este vacío, optamos por crear un instrumento en español, usando una metodología mixta y una aproximación inductiva. El objetivo fue desarrollar, a partir de las percepciones de estudiantes y docentes chilenos, un cuestionario ad hoc para el aprendizaje clínico, lingüística y culturalmente apropiado a la realidad nacional. El presente artículo describe el desarrollo y validación del Cuestionario de Estrategias para el Aprendizaje de la Clínica (CEACLIN) y sus características psicométricas en una muestra de estudiantes chilenos.

\section{Materiales y Métodos}

Esta investigación contó con la aprobación del Comité de Ética de la Escuela de Medicina de la Pontificia Universidad Católica de Chile, y del Fondo Nacional de Desarrollo Científico y Tecnológico (FONDECYT).

\section{Desarrollo del instrumento}

El proceso constó de 4 etapas: 1) confección de un borrador en base a percepciones de estudiantes y docentes sobre cómo aprenden la clínica los estudiantes de medicina ${ }^{7} ; 2$ ) análisis de este borrador por un panel nacional de expertos mediante la técnica Delfi para lograr acuerdos; 3 ) pilotaje del cuestionario resultante; 4) aplicación masiva y 5 ) estudio de confiabilidad y validez (Figura 1).

El primer borrador (80 ítems) se construyó a partir de las 10 categorías temáticas derivadas de la codificación abierta de 8 grupos focales de estudiantes y 8 entrevistas a docentes clínicos de la escuela de medicina de la Pontificia Universidad Católica de Chile (EMPUC) ${ }^{7}$. Estas categorías temáticas se validaron mediante el acuerdo intersubjetivo entre los investigadores: A partir de ellas, se construyeron los 80 ítems iniciales del primer borrador mediante un proceso de análisis iterativo en el que participaron todos los miembros del equipo de investigación. Los grupos focales y entrevistas, y el análisis cualitativo de ellos se realizaron durante 2012; esto se encuentra descrito in extenso en un artículo previo ${ }^{7}$.

Para conformar un panel de expertos de representación nacional, se invitaron por vía electrónica a docentes y estudiantes de las 18 escuelas de medicina que contaban con acreditación vigente el año 2013. Éstos fueron seleccionados por recomendación ya sea de autoridades de las distintas escuelas y Centros de alumnos, o por docentes y estudiantes activos en educación médica. Los criterios de inclusión para los docentes fueron: hombres y mujeres con al menos cinco años de experiencia en docencia clínica de pregrado. Para los estudiantes, hombres y mujeres que estuvieran cursando entre tercer y sexto año de medicina. El retorno de las invitaciones fue superior a $70 \%$. El panel quedó constituido por 42 estudiantes y 


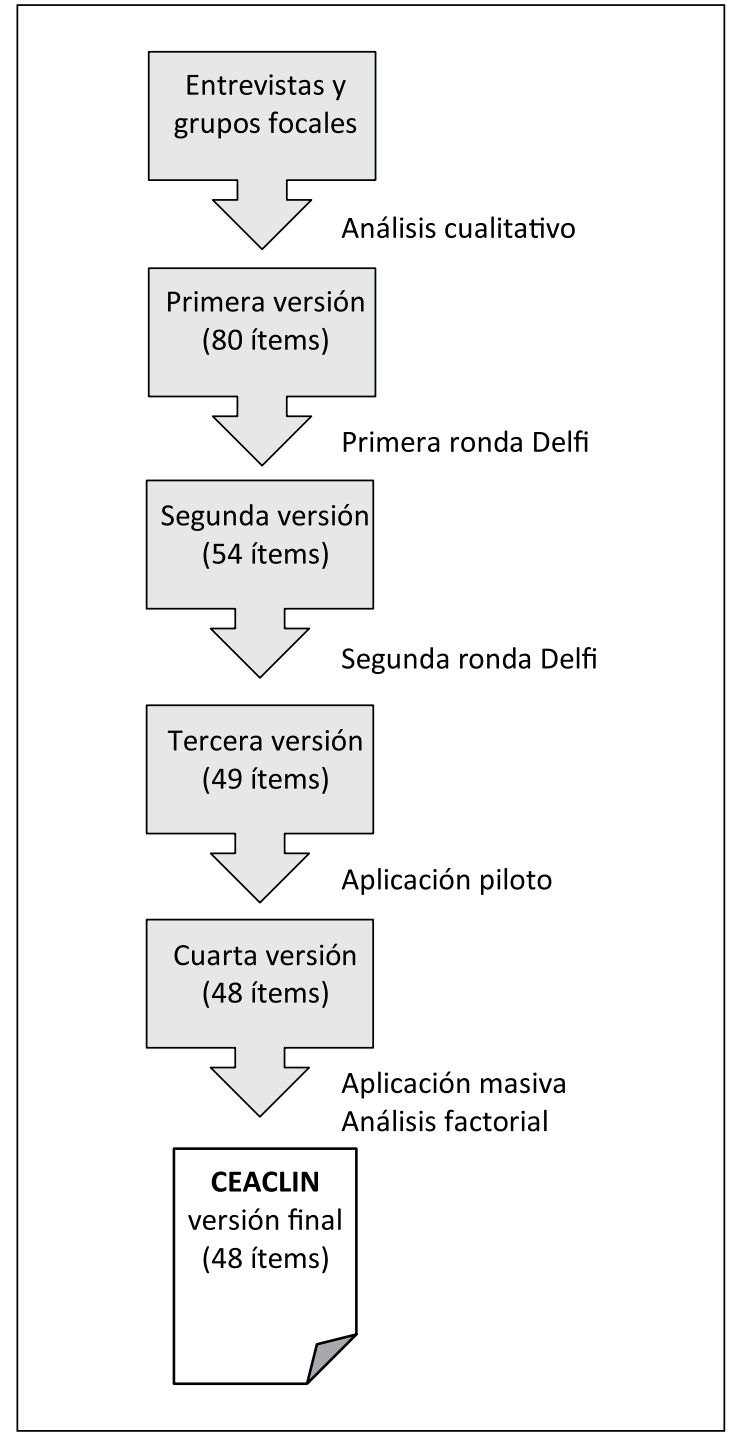

Figura 1. Diagrama de flujo del desarrollo del Cuestionario de Estrategias de Aprendizaje de la Clínica (CEACLIN).

49 docentes clínicos de 15 escuelas de medicina: Universidad Austral de Chile, Universidad Católica del Maule, Universidad Católica del Norte, Universidad de Antofagasta, Universidad de Chile, Universidad de Concepción, Universidad del Desarrollo, Universidad de Los Andes, Universidad de Santiago de Chile, Universidad de Valparaíso, Universidad Diego Portales, Universidad Finis Terrae, Universidad Mayor, Universidad Nacional Andrés Bello y Pontificia Universidad Católica de Chile (PUC). Además, participaron dos expertos en psicometría y dos secretarias encargadas de los cursos preclínicos de la EMPUC, con estrecho contacto y amplio conocimiento de los estudiantes.

Durante 2013, el panel realizó dos rondas de análisis usando la técnica Delfi para alcanzar acuerdos $^{18,19}$. Los miembros recibieron por vía electrónica el borrador de 80 ítems con la instrucción de evaluar la pertinencia y claridad de sus ítems. Al cabo de esta ronda, el cuestionario se redujo a 54 items, considerados importantes o muy importantes ( $\geq 3$ puntos de una escala de 4 puntos). En la segunda ronda, los panelistas recibieron la nueva versión junto a un informe de los resultados generales de la primera. La tasa de respuesta fue $71 \%$ estudiantes y $74 \%$ docentes. En base a los resultados se eliminaron cinco ítems, generándose la tercera versión (49 items). No se realizaron rondas adicionales por considerar que se había alcanzado la saturación del proceso.

Este cuestionario se administró a 29 estudiantes de medicina de la EMPUC, quienes lo contestaron con una escala de frecuencia tipo Likert de 0 a 4 , en la que: $0=$ no aplica/no entiendo, $1=$ casi nunca, $2=$ algunas veces, $3=$ con frecuencia $y$ $4=$ casi siempre. En base a los resultados del piloto se eliminó un ítem. Así, se obtuvo la versión final, llamada Cuestionario de Estrategias de Aprendizaje de la Clínica (CEACLIN) (Figura 1).

\section{Estudio de confiabilidad y validez}

CEACLIN se administró a 336 estudiantes de medicina de la PUC que cursaban entre cuarto y sexto año durante el segundo semestre de 2013. La confiabilidad del instrumento se evaluó con el coeficiente $\alpha$ de Cronbach $^{20}$ y la validez de constructo mediante un análisis factorial exploratorio seguido de una rotación varimax ${ }^{21}$. Para determinar el número de factores se realizó un análisis paralelo (parallel analysis) $^{22,23}$. Los análisis se realizaron usando el paquete psych de R, y el software SPSS Inc. Released 2008. SPSS Statistics for Windows, Version 17.0. Chicago: SPSS Inc.

\section{Resultados}

El instrumento final, denominado CEACLIN, contiene 48 ítems que describen estrategias para aprender la clínica (Tabla 1). Los estadígrafos de la aplicación a 336 estudiantes se presentan también en la Tabla 1. Los promedios oscilaron entre 1,5 


\section{Tabla 1. Ítems y estadígrafos del CEACLIN}

\section{Cuestionario de Estrategias de Aprendizaje de la Clínica (CEACLIN) (escala: 1: casi nunca, 2: a veces, 3: frecuentemente, 4: casi siempre)}

\section{Ítems}

$\% \mathbf{R} \quad \overline{\mathrm{X}}+\mathrm{DE}$

1. Para aprender más durante la rotación, pido feedback sobre mi desempeño a mis tutores/docentes $\quad 100 \quad 2,7 \pm 1,0$

2. Para aprender mejor los contenidos teóricos de este año, interactúo con mis compañeros (estudiamos juntos, conversamos, nos enseñamos mutuamente, etc.)

$100 \quad 3,0 \pm 0,9$

3. Para mantenerme motivado(a) en la carrera, necesito hacer otras cosas que también me gustan (p. ej. deportes, artes, vida social, etc.)

$100 \quad 3,5 \pm 0,8$

4. Cuando estoy con mucha carga académica y me falta tiempo para estudiar, postergo el descanso y/o sacrifico parte de mis horas de sueño

5. Cuando tengo alguna duda respecto de un paciente o procedimiento, primero estudio por mi cuenta y luego pido ayuda, si es necesario

6. Las notas son un estímulo importante para mantenerme motivado(a) en la carrera

$2,9 \pm 0,8$

$100 \quad 3,0 \pm 0,9$

7. Para enfrentar mejor la rotación, me informo sobre mi rol y reglas del lugar en que me desempeñaré $\quad 100 \quad 2,9 \pm 1,0$

8. Busco otras instancias de aprendizaje (seminarios, congresos, cursos, etc.) para aprender mejor los contenidos teóricos de este año

9. Cuando tengo alguna duda respecto de un paciente (en la entrevista o procedimiento), le pregunto a mis compañeros

$99,7 \quad 2,1 \pm 0,9$

$100 \quad 3,3 \pm 0,7$

10. Organizo y planifico mis actividades de la semana

$99,7 \quad 2,8 \pm 1,0$

11. Cuando cometo errores o no sé responder una pregunta frente al grupo, me dan ganas de estudiar más

12. Cuando estoy agobiado(a) por la carga académica/asistencial, le cuento a mis amigos o familiares

$99,7 \quad 3,2 \pm 0,8$

$99,7 \quad 3,0 \pm 1,0$

13. Trato de ver al mayor número de pacientes posible para aprender más durante la rotación

$99,7 \quad 2,8 \pm 0,8$

14. Cuando tengo alguna duda respecto de un paciente (en la entrevista y/o procedimiento), recurro al residente o al interno

$99,7 \quad 3,2 \pm 0,9$

15. Para aprender más, pongo atención a la manera en que mis tutores se comunican con los pacientes y sus familiares

$100 \quad 3,4 \pm 0,7$

16. Para aprender mejor los contenidos teóricos de este año, consulto muchas fuentes de información (apuntes, power points, vídeos, páginas web, manuales)

17. Para funcionar mejor durante la rotación, me pongo de acuerdo con mis compañeros(as) de grupo para decidir cómo trabajar

$99,4 \quad 2,6 \pm 1,0$

18. Cuando las actividades no están bien organizadas o no son claras, me esfuerzo para mantener las ganas de aprender

19. Aprendo mejor los contenidos cuando hago una lista de los temas que no he entendido, para revisarlos después

$100 \quad 2,6 \pm 0,9$

$99,7 \quad 2,6 \pm 1,1$

20. Cuando tengo alguna duda respecto de un paciente (en la entrevista y/o procedimiento), le pregunto a mi tutor(a)

21. Para aprender mejor, busco ambientes de confianza y tolerancia

$100 \quad 3,5 \pm 0,7$

$99,4 \quad 3,7 \pm 0,6$

22. Me es más fácil aprender la clínica cuando -además del razonamiento- integro otras habilidades como por ejemplo, capacidad de escucha activa, sentido del humor, etc

$100 \quad 3,7 \pm 0,6$

23. Me motiva aprender cuando hay momentos de reflexión posteriores a una visita o discusión de un caso clínico

$100 \quad 3,5 \pm 0,7$

24. Cuando tengo dudas respecto de un paciente (en la entrevista y/o procedimiento), pregunto a alguien del equipo de salud (matrona, enfermera, etc.)

25. Para aprender mejor los contenidos de este año, hago resúmenes y/o mapas conceptuales 
26. Para comprobar mi aprendizaje, comparo la información que obtuve en la entrevista clínica con la que aparece en la ficha del paciente

27. Para aprender mejor los contenidos teóricos de este año, comparto con mis compañeros los apuntes por medios digitales (dropbox, googledrive, mail, facebook, etc.)

28. Para establecer una buena relación con el paciente durante la entrevista clínica, pongo atención a sus emociones

29. Busco oportunidades adicionales a las entregadas por la escuela para practicar lo aprendido en la rotación (p. ej. voluntariado, operativos de salud)

30. Además de estudiar de los apuntes, consulto el material sugerido por el profesor (libros, manuales, artículos)

31. Para aprender más durante la rotación, observo cómo lo hacen mis compañeros(as)

32. Al estudiar, selecciono la materia de estudio o tópicos que me parecen más relevantes

33. Cuando me dan más autonomía en el manejo de mis pacientes o actividades, aumentan mis ganas de aprender

34. Cuando estoy con mucha carga académica/asistencial, privilegio estudiar por mi cuenta por sobre asistir a clases

35. Ver pacientes me ayuda para mantenerme motivado(a) en la carrera

36. Para que el paciente confíe profesionalmente en mí, intento aumentar mi seguridad durante la entrevista

37. Al estudiar, reviso toda la materia

$99,4 \quad 2,5 \pm 1,2$

38. Cuando estoy agobiado(a) por la carga académica o por mi rendimiento, uso medicamentos (ansiolíticos, estimulantes, etc.) que me ayuden a funcionar mejor

39. Cuando en la entrevista y/o procedimiento algo no me resulta bien, informo al tutor

$99,7 \quad 3,6 \pm 0,7$

$100 \quad 3,0 \pm 0,9$

40. Cuando mis tutores, pares y/o pacientes me dicen que lo he hecho bien, me motivo a aprender más

41. Intento anticipar las preguntas de las pruebas teóricas, como método de estudio

$99,7 \quad 1,6+1,0$

42. Cuando la carga académica me hace sentir que necesito ayuda médica o psicológica, la busco

$100 \quad 3,0 \pm 0,9$

$99,7 \quad 3,7 \pm 0,6$

$100 \quad 2,9 \pm 1,0$

43. Para aprender mejor los contenidos teóricos de este año, uso pocas fuentes de información, pero confiables

$98,5 \quad 2,0 \pm 1,1$

$99,4 \quad 3,1 \pm 0,9$

44. Cuando estoy agobiado(a) por la carga académica, consumo alcohol y/u otras drogas de recreación

$99,7 \quad 1,5+0,9$

45. Me es más fácil aprender la clínica durante la rotación cuando asumo responsabilidades relativas al paciente

$99,7 \quad 3,5 \pm 0,7$

46. Cuando estoy con mucha carga académica, postergo mis actividades personales (recrearme, salir con amigos o familia)

47. Para evitar equivocarme con el paciente, trato de recordar mis errores anteriores para no repetirlos $\quad 100 \quad 3,6 \pm 0,6$

48. Para establecer una buena relación con el paciente durante la entrevista clínica, pongo atención a las emociones que me surgen

X̃: promedio, DE: desviación estándar, \% R: porcentaje de respuestas de la aplicación a 336 estudiantes de medicina.

y 3,7 en una escala de 4 puntos (1: casi nunca; 4 : casi siempre). El porcentaje de respuesta de todos los ítems superó 99\% (Tabla 1).

El análisis indicó que CEACLIN es altamente confiable $(\alpha=0,84)$, comparado con los valores considerados aceptables ${ }^{23}$. Por otra parte, el análisis factorial, seguido por una rotación varimax y un análisis paralelo, reveló una estructura interna de 11 factores, cuyos autovalores se presenta en la Tabla 2. Estos factores en conjunto dan cuenta de $48 \%$ de la varianza. Los ítems del instrumento fueron asignados al factor donde presentaban mayor carga factorial. La distribución de los items por factor se detalla en la Tabla 3. 
Tabla 2. Valores propios de los factores y contribución a la varianza

\begin{tabular}{|cccc|}
\hline Factor & \multicolumn{3}{c|}{ Valores propios iniciales } \\
Total & $\begin{array}{c}\text { \% de la } \\
\text { varianza }\end{array}$ & $\begin{array}{c}\text { \% } \\
\text { acumulado }\end{array}$ \\
\hline 1 & 6,751 & 14,065 & 14,065 \\
\hline 2 & 2,428 & 5,058 & 19,123 \\
\hline 3 & 2,162 & 4,503 & 23,626 \\
\hline 4 & 1,825 & 3,802 & 27,428 \\
\hline 5 & 1,744 & 3,633 & 31,061 \\
\hline 6 & 1,573 & 3,278 & 34,339 \\
\hline 7 & 1,511 & 3,147 & 37,486 \\
\hline 8 & 1,364 & 2,841 & 40,327 \\
\hline 9 & 1,329 & 2,768 & 43,095 \\
\hline 10 & 1,308 & 2,725 & 45,820 \\
\hline 11 & 1,225 & 2,551 & 48,372 \\
\hline
\end{tabular}

Los factores fueron nombrados como se detalla a continuación, por consenso de los miembros del equipo de investigación, luego de un análisis iterativo. Factor 1: Autonomía, factor 2: Resolver dudas y problemas, factor 3: Buscar y organizar la información, factor 4: Proactividad, factor 5: Recurrir a los demás, factor 6: Atender a las emociones, factor 7: Buscar lo confiable, factor 8: Evadirse del agobio, factor 9: Afrontar el agobio, factor 10: Motivación, factor 11: Postergar lo personal.

\section{Discusión}

Este artículo describe el desarrollo y validación del Cuestionario de Estrategias de Aprendizaje de la Clínica (CEALIN). Este proceso incluyó: 1) generación de un borrador a partir de una indagación cualitativa; 2) validación por un panel Delfi de estudiantes y docentes de 15 escuelas de medicina chilenas; 3) aplicación piloto; 4) aplicación masiva y 5) evaluación de confiabilidad y validez de constructo del instrumento resultante.

El análisis psicométrico -basado en las respuestas de 336 estudiantes- indica que CEALIN es un instrumento altamente confiable (Cronbach $\alpha=0,84$ ), compuesto por 11 factores: Autonomía, Resolver dudas y problemas, Buscar y organizar información, Proactividad, Recurrir a otros, Atender a las emociones, Buscar lo confiable, Evadirse del Agobio, Enfrentar el agobio, Motivación y Postergar lo personal.

Para visualizar más claramente las relaciones entre estos factores, proponemos un modelo que los agrupa en cuatro dimensiones teóricas: Involucramiento académico (factores $1,4,5,10$ ), Técnicas de estudio (factores 2 y 3 ), Regulación emocional (factores 6 y 7) y Manejo del agobio (factores 8, 9 y 11) (Figura 2). Mirado desde esta

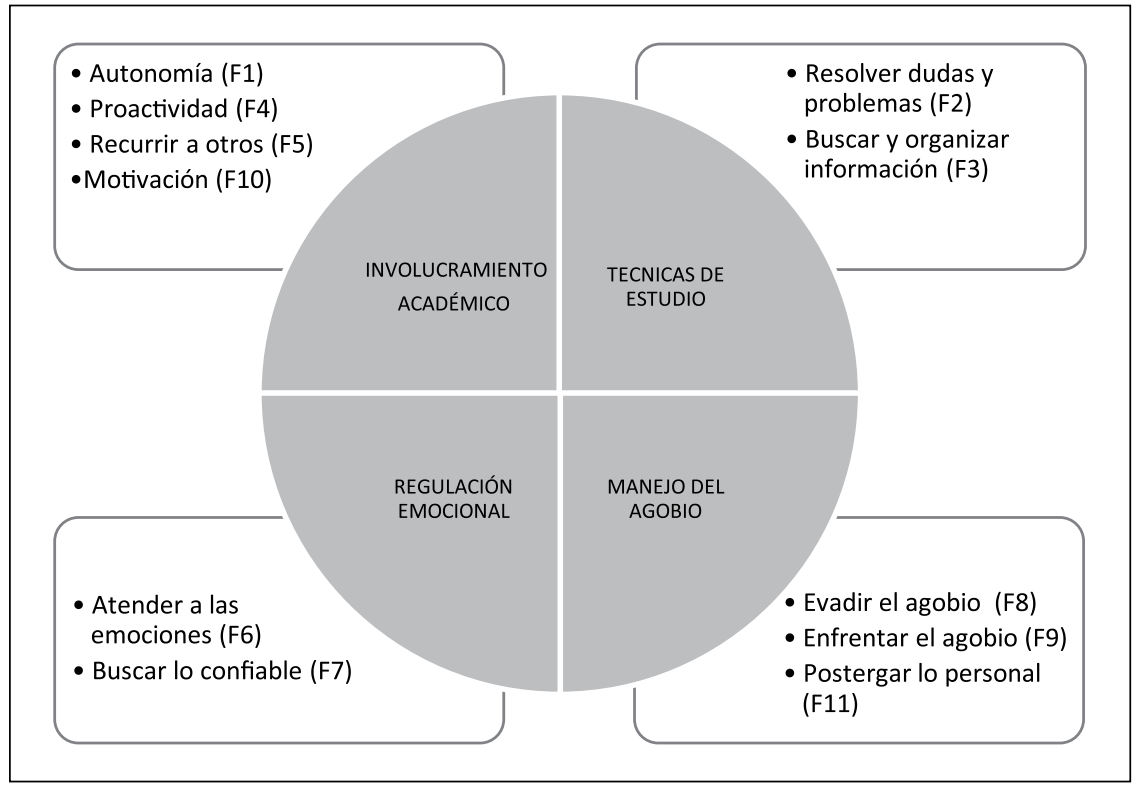

Figura 2. Propuesta de clasificación de los factores de CEACLIN en categorías conceptuales. 
Tabla 3. Distribución de los ítems por factor

\begin{tabular}{|c|c|c|c|c|c|c|c|c|c|c|c|}
\hline \multicolumn{12}{|c|}{ Factores } \\
\hline ítem & F1 & F2 & F3 & F4 & F5 & F6 & F7 & F8 & F9 & F10 & F11 \\
\hline 35 & 0,81 & $-0,07$ & $-0,06$ & 0,41 & 0,17 & 0,21 & 0,01 & $-0,26$ & 0,12 & $-0,02$ & $-0,10$ \\
\hline 33 & 0,66 & 0,07 & 0,19 & 0,09 & 0,01 & 0,12 & 0,13 & 0,12 & $-0,09$ & 0,15 & $-0,09$ \\
\hline 45 & 0,66 & 0,21 & 0,02 & 0,06 & $-0,04$ & 0,13 & 0,14 & 0,13 & $-0,06$ & 0,15 & 0,03 \\
\hline 36 & 0,63 & 0,04 & 0,15 & 0,16 & 0,16 & 0,12 & 0,24 & $-0,10$ & $-0,09$ & $-0,01$ & 0,10 \\
\hline 47 & 0,32 & 0,18 & 0,05 & 0,18 & 0,28 & 0,19 & 0,17 & 0,11 & $-0,17$ & $-0,06$ & 0,15 \\
\hline 14 & 0,15 & 0,68 & 0,09 & 0,07 & $-0,07$ & 0,13 & 0,12 & 0,03 & 0,05 & 0,12 & 0,11 \\
\hline 17 & 0,07 & 0,53 & 0,22 & 0,14 & 0,22 & 0,09 & 0,01 & 0,19 & $-0,01$ & 0,02 & $-0,11$ \\
\hline 24 & $-0,06$ & 0,44 & 0,12 & 0,17 & 0,12 & 0,02 & $-0,05$ & 0,05 & 0,06 & $-0,01$ & 0,01 \\
\hline 39 & 0,11 & 0,40 & 0,04 & $-0,18$ & 0,22 & 0,16 & 0,05 & $-0,05$ & 0,10 & 0,25 & 0,00 \\
\hline 15 & 0,16 & 0,39 & 0,18 & 0,03 & 0,34 & 0,15 & 0,27 & $-0,26$ & 0,09 & $-0,08$ & 0,08 \\
\hline 9 & $-0,04$ & 0,29 & $-0,16$ & 0,17 & 0,27 & 0,09 & 0,14 & 0,25 & $-0,08$ & 0,06 & 0,05 \\
\hline 30 & 0,01 & $-0,03$ & 0,65 & 0,11 & 0,21 & 0,11 & $-0,23$ & $-0,03$ & 0,09 & 0,11 & 0,10 \\
\hline 16 & 0,00 & 0,14 & 0,51 & 0,10 & 0,07 & 0,14 & $-0,07$ & $-0,23$ & $-0,02$ & $-0,02$ & 0,18 \\
\hline 8 & 0,14 & 0,18 & 0,44 & 0,39 & $-0,04$ & 0,00 & $-0,12$ & 0,24 & 0,06 & 0,03 & 0,13 \\
\hline 19 & 0,04 & 0,28 & 0,42 & 0,14 & $-0,03$ & 0,04 & 0,28 & $-0,07$ & 0,04 & 0,25 & $-0,06$ \\
\hline 32 & 0,26 & 0,21 & 0,39 & $-0,12$ & 0,15 & $-0,04$ & 0,38 & 0,15 & $-0,08$ & $-0,04$ & $-0,03$ \\
\hline 7 & 0,12 & 0,31 & 0,39 & 0,05 & 0,03 & 0,08 & 0,16 & 0,06 & 0,04 & 0,13 & $-0,05$ \\
\hline 1 & 0,09 & 0,24 & 0,34 & 0,11 & 0,04 & 0,04 & 0,06 & 0,08 & $-0,01$ & 0,08 & $-0,15$ \\
\hline 5 & 0,08 & $-0,05$ & 0,32 & 0,18 & $-0,20$ & $-0,10$ & 0,11 & $-0,01$ & 0,15 & 0,00 & 0,31 \\
\hline 25 & 0,13 & 0,13 & 0,30 & 0,26 & $-0,12$ & 0,08 & 0,18 & $-0,05$ & 0,02 & 0,21 & 0,07 \\
\hline 10 & 0,05 & 0,06 & 0,12 & 0,52 & $-0,03$ & 0,00 & 0,16 & $-0,07$ & $-0,03$ & 0,21 & 0,03 \\
\hline 18 & 0,15 & 0,35 & 0,17 & 0,46 & 0,03 & 0,09 & 0,02 & $-0,14$ & $-0,03$ & $-0,01$ & $-0,02$ \\
\hline 29 & 0,12 & 0,13 & 0,14 & 0,43 & 0,03 & 0,20 & $-0,11$ & 0,31 & 0,05 & $-0,15$ & 0,02 \\
\hline 12 & $-0,07$ & 0,08 & $-0,02$ & 0,37 & 0,26 & 0,24 & 0,27 & 0,01 & 0,12 & 0,21 & $-0,02$ \\
\hline 13 & 0,25 & 0,01 & 0,24 & 0,35 & 0,24 & 0,02 & $-0,09$ & $-0,12$ & 0,00 & $-0,08$ & 0,11 \\
\hline 37 & 0,17 & $-0,01$ & $-0,01$ & 0,35 & 0,12 & 0,02 & $-0,09$ & $-0,12$ & $-0,04$ & 0,06 & 0,15 \\
\hline 11 & 0,11 & 0,14 & 0,17 & 0,31 & 0,08 & $-0,07$ & 0,08 & $-0,04$ & 0,02 & 0,18 & 0,13 \\
\hline 2 & 0,08 & 0,19 & 0,08 & 0,27 & 0,20 & 0,08 & 0,13 & 0,10 & $-0,08$ & 0,07 & $-0,09$ \\
\hline 31 & $-0,02$ & 0,14 & 0,08 & 0,14 & 0,63 & 0,07 & 0,17 & $-0,01$ & 0,03 & 0,05 & $-0,01$ \\
\hline 23 & 0,33 & 0,06 & 0,14 & $-0,04$ & 0,56 & 0,17 & $-0,12$ & $-0,09$ & $-0,08$ & 0,07 & $-0,03$ \\
\hline 27 & 0,04 & 0,04 & 0,02 & 0,04 & 0,39 & 0,13 & 0,22 & 0,31 & $-0,11$ & 0,17 & 0,05 \\
\hline 20 & 0,17 & 0,27 & $-0,09$ & 0,14 & 0,37 & $-0,01$ & 0,17 & $-0,23$ & 0,02 & 0,05 & 0,25 \\
\hline 48 & 0,19 & 0,26 & 0,10 & 0,17 & 0,14 & 0,73 & 0,06 & 0,08 & $-0,08$ & 0,10 & $-0,01$ \\
\hline 28 & 0,35 & 0,13 & 0,12 & $-0,03$ & 0,11 & 0,72 & 0,18 & $-0,02$ & 0,13 & 0,05 & 0,03 \\
\hline 26 & 0,16 & 0,15 & 0,06 & 0,15 & 0,16 & 0,30 & 0,22 & 0,06 & 0,08 & $-0,03$ & 0,25 \\
\hline 3 & 0,14 & 0,01 & 0,11 & $-0,01$ & 0,15 & 0,25 & 0,14 & 0,14 & 0,06 & $-0,14$ & $-0,07$ \\
\hline 21 & 0,13 & $-0,01$ & 0,06 & 0,06 & 0,20 & 0,23 & 0,55 & $-0,18$ & 0,14 & 0,11 & $-0,03$ \\
\hline 43 & 0,11 & 0,07 & $-0,07$ & 0,02 & 0,02 & 0,04 & 0,44 & 0,02 & 0,01 & 0,05 & $-0,01$ \\
\hline 22 & 0,34 & $-0,03$ & $-0,01$ & 0,04 & 0,29 & 0,18 & 0,44 & 0,15 & 0,06 & 0,07 & 0,18 \\
\hline 44 & $-0,08$ & 0,05 & 0,06 & $-0,02$ & 0,04 & 0,04 & $-0,07$ & 0,69 & 0,17 & $-0,03$ & 0,04 \\
\hline 34 & 0,07 & 0,05 & $-0,10$ & $-0,12$ & $-0,05$ & 0,01 & 0,03 & 0,39 & 0,12 & $-0,02$ & 0,07 \\
\hline 38 & $-0,09$ & 0,00 & 0,11 & $-0,21$ & $-0,02$ & $-0,05$ & 0,06 & 0,33 & 0,88 & 0,07 & 0,20 \\
\hline 42 & $-0,11$ & 0,22 & 0,04 & 0,17 & $-0,04$ & 0,18 & 0,12 & 0,15 & 0,54 & 0,10 & $-0,10$ \\
\hline 40 & 0,42 & 0,19 & 0,05 & 0,09 & 0,12 & 0,30 & 0,21 & $-0,15$ & $-0,12$ & 0,61 & 0,19 \\
\hline 6 & 0,02 & 0,05 & 0,10 & 0,12 & 0,02 & $-0,04$ & 0,02 & $-0,03$ & 0,09 & 0,43 & 0,19 \\
\hline 41 & 0,13 & 0,08 & 0,17 & 0,13 & 0,29 & 0,02 & 0,08 & 0,14 & 0,10 & 0,43 & $-0,09$ \\
\hline 4 & $-0,07$ & $-0,14$ & 0,01 & 0,08 & 0,03 & $-0,04$ & 0,06 & 0,13 & 0,02 & 0,03 & 0,65 \\
\hline 46 & 0,03 & 0,11 & 0,06 & 0,02 & 0,01 & 0,06 & $-0,08$ & 0,01 & $-0,01$ & 0,12 & 0,46 \\
\hline
\end{tabular}


perspectiva, parece razonable plantear que, para aprender la clínica, el estudiante de medicina debe contar estrategias efectivas para mantenerse involucrado (engaged), técnicas de estudio apropiadas para aprender de la experiencia, regulación emocional y estrategias de autocuidado y manejo del estrés.

Aunque el CEACLIN no fue desarrollado en base a ninguna teoría; reconocemos en él elementos de las teorías socio-culturales del aprendizaje $^{25-27}$ y la Teoría de Autodeterminación ${ }^{28}$. El componente social aparece en 13 de los 48 ítems que describen formas de aprender con y de otros, ya sean compañeros tutores, residentes, docentes, pacientes o miembros del equipo de salud (en la Escuela de Medicina de la PUC, estos grupos están compuestos usualmente por 5 a 6 estudiantes de $4^{\circ}$ o $5^{\circ}$ año, uno o dos internos, un residente y un tutor clínico, que realizan rotaciones diarias en conjunto por un lapso variable que va entre 2 y 8 semanas). Como ejemplo, el ítem 14: "Cuando tengo alguna duda respecto de un paciente (en la entrevista $y /$ o procedimiento), recurro al residente o al interno".

Esta sensibilidad del CEACLIN a los aspectos sociales del aprendizaje puede estar relacionada con la exposición a la docencia clínica en grupos pequeños, donde los jóvenes aprenden junto a estudiantes de distintos niveles, guiados por un tutor, en el contexto del cuidado del enfermo. Esto se parece en cierta medida a las comunidades de práctica propuestas por Wenger ${ }^{27}$, que describen a grupos heterogéneos de personas que aprenden unos de otros y trabajan en pos de un objetivo común.

Además de los aspectos sociales, el CEACLIN indaga acerca de la motivación, en una manera consistente con la Teoría de Autodeterminación de Deci y Ryan ${ }^{28}$. Ésta postula que un individuo se sentirá motivado por aprender (o realizar una tarea) en la medida en que cuente con cierta autonomía (ver ítem 33 del CEACLIN), logre un grado de autoeficacia (ítem 40) y mantenga una conexión significativa con otros (ítem 2).

Los factores 8,9 y 11 del CEACLIN aluden al manejo del estrés y describen conductas de evasión, enfrentamiento del agobio, y postergación personal, respectivamente. Ejemplos de estas conductas pueden encontrarse en los ítems 44, 42 y 46 (Tabla 1).

Es sabido que en Chile y otros países, los es- tudiantes de medicina postergan sus necesidades personales y de descanso por estudiar; dejan de practicar hobbies y cultivar sus intereses en un intento de responder a las exigencias académicas ${ }^{7}$, incluso pueden ir más allá incurriendo en conductas de riesgo. Esto suele tener consecuencias negativas sobre la salud, el desempeño académi$\mathrm{CO}^{29-33}$ y también el profesionalismo ${ }^{34}$. La aparición del tema de cómo manejar el agobio en un cuestionario de estrategias de aprendizaje generado inductivamente, como es el CEACLIN, subraya la importancia que otorgan los propios estudiantes al bienestar para el aprendizaje.

Una de las fortalezas del CEACLIN deriva de su construcción: sus ítems provienen de la experiencia concreta de los propios estudiantes y sus docentes clínicos, lo que lo hace culturalmente pertinente a la formación de médicos en nuestro país. Otro hecho a destacar es que el CEACLIN fue validado por un panel Delfi de amplia representatividad nacional ( $83 \%$ de las escuelas de medicina acreditadas a la fecha del estudio). Esto subraya su validez y hace razonable pensar que este instrumento será de utilidad para los estudiantes y docentes de muchas escuelas de medicina del país.

Otra fortaleza es que la validación de la estructura interna del CEACLIN se basó en las respuestas de prácticamente todo el universo de estudiantes que cursan entre 4 y $6^{\circ}$ año de medicina en la PUC (336 de 360; 93\%), lo que asegura la representatividad de los resultados.

¿Cómo expresar los resultados del CEACLIN? ¿Mediante el puntaje global, los puntajes por factor o por categoría conceptual? Pensamos que para tomar esta decisión es preciso conocer la correlación de estos parámetros con variables relevantes para la vida de los estudiantes como su rendimiento académico y bienestar subjetivo (tema que es motivo de una investigación en curso). Dicho esto, parece evidente que los valores por categoría conceptual serán más informativos que el score global y probablemente más sólidos que los puntajes de aquellos factores conformados por menos de tres ítems ${ }^{35}$. Por otra parte, el uso de los dominios hará más fácil deducir un significado de las mediciones pues acota el desafío del aprendizaje clínico a cuatro ámbitos. El estudiante debe: 1) estar equipado con estrategias efectivas para mantener su motivación e involucramiento; 2) desarrollar técnicas de estudio apropiadas para aprender de las experiencias concretas; 3 ) conocer 
y regular sus emociones en contextos de aprendizaje y 4) contar con estrategias de autocuidado y manejo del estrés (Figura 2).

A diferencia de otros cuestionarios como el Study Process Questionnaire (SPQ) ${ }^{36}$, que clasifica a los estudiantes de acuerdo a si tienen una aproximación profunda o superficial al estudio; o el Inventario de Estilos de Aprendizaje de $\mathrm{Kolb}^{11}$, que los distingue según su modo preferente de procesar la información, CEACLIN informa al estudiante de su repertorio actual de conductas para aprender en situaciones específicas, sin etiquetarlo a priori, y le da información útil para mejorar. Esto supone una ventaja notable. A un estudiante clasificado como 'superficial' en el cuestionario SPQ o como 'Asimilador' en el Kolb, le sería difícil cambiar rápidamente de categoría. Sin embargo, un estudiante que no suele pedir feedback a su tutor (ítem 1 del CEACLIN), puede intentar hacerlo más a menudo.

Así, los estudiantes pueden usar la información que proporciona el CEACLIN como herramienta de autoconocimiento y cambio; pueden revisar continuamente sus estrategias y eventualmente modificarlas para potenciar su desempeño académico y bienestar integral. Esto sería consistente con el concepto de 'study orchestration', que plantea que los estudiantes usan las estrategias de manera flexible, de acuerdo a lo que es más adecuado al ambiente y contexto de aprendizaje en que están ${ }^{37}$.

\section{Limitaciones del estudio}

La fiabilidad y validez del CEACLIN se determinaron mediante el coeficiente $\alpha$ de Chronbach, y el análisis factorial, respectivamente. Aunque la mayor parte de los estudios utiliza sólo estos dos indicadores, sería interesante evaluar en estudios futuros otros índices de fiabilidad y validez (p. e. fiabilidad intraobservador y validez concurrente), de modo de hacer una caracterización exhaustiva de las propiedades psicométricas de CEACLIN.

En cuanto a su desarrollo, una de las posibles limitaciones a la generalizibilidad del CEACLIN es que el borrador inicial recogió las percepciones de estudiantes y docentes de una sola escuela de medicina. Este sesgo no tendría relación con los temas que CEACLIN aborda (que fueron refrendados por el panel nacional), sino con 'lo que no pregunta' y -por ende- permanece invisible. Es posible, entonces, que existan conductas no ex- ploradas por este instrumento que pudieran ser relevantes para el aprendizaje. Esta posibilidad debe ser abordada en el futuro, mediante estudios cualitativos multicéntricos.

El tamaño de la muestra usada en este estudio estuvo limitado por el tamaño de la población respectiva; participaron 336 los 360 estudiantes (93\%) que cursaban $4^{\circ}$ a $6^{\circ}$ año de medicina en la PUC. Esto dio una relación de 7:1 (participantes/ número de ítems del cuestionario), valor considerado bajo por algunos autores ${ }^{38}$, pero suficiente por otros como Gorsuch (1983) que recomienda una proporción de cinco participantes por variable medida ${ }^{39}$. Por otra parte, en una revisión reciente $^{40}$ (Lloret-Segura et al, 2014) se estima que un tamaño muestral de 300 es adecuado para la mayor parte de los análisis descriptivos y psicométricos de los ítems. Dada esta controversia, en estudios futuros sería importante contar con tamaños muestrales mayores.

Otra de las limitaciones tiene que ver con que la varianza total explicada por los 11 factores resultó menor de 50\%. Esto, junto con el elevado número de factores, indica que estamos ante un fenómeno complejo, multifactorial, del que quizás están quedando fuera algunos aspectos. A este respecto, sería importante realizar un análisis factorial confirmatorio con una muestra de participantes diversa y más numerosa, que permita verificar la estabilidad de la estructura factorial encontrada.

En conclusión, CEACLIN es un instrumento válido, confiable y de fácil uso que permite estudiar un fenómeno complejo y muy relevante para la formación de los futuros médicos. CEACLIN permite identificar las estrategias y aproximaciones que usan los estudiantes de medicina chilenos para aprender la clínica. En nuestro conocimiento, es el único instrumento validado en español, diseñado con este propósito.

Esperamos que sea de utilidad a los estudiantes para revisar y mejorar sus estrategias de aprendizaje. Pensamos también que este instrumento puede proporcionar una información valiosa a educadores médicos y autoridades para revisar las prácticas docentes e incorporar instancias curriculares tempranas para que los estudiantes 'aprendan a aprender de la práctica', mejoren sus habilidades relacionales y aprendan a cuidar de sí mismos; todas ellas, habilidades necesarias para el aprendizaje clínico. 
Agradecimientos: Agradecemos a todos los estudiantes y docentes que formaron parte del panel de expertos y a las autoridades respectivas de: Universidad Austral de Chile, Universidad Católica del Maule, Universidad Católica del Norte, Universidad de Antofagasta, Universidad de Chile, Universidad de Concepción, Universidad del Desarrollo, Universidad de Los Andes, Universidad de Santiago de Chile, Universidad de Valparaíso, Universidad Diego Portales, Universidad Finis Terrae, Universidad Mayor, Universidad Andrés Bello y Pontificia Universidad Católica de Chile.

\section{Referencias}

1. Sánchez I, Riquelme A, Moreno R, Mena B, Dagnino J, Grebe G. Revitalizing medical education: the school of medicine at the Pontificia Universidad Católica de Chile. Clin Teacher 2008; 5: 57-61.

2. Martinic S, Moreno R, Muller M, Pimentel, F. Ritthershaussen, Calderón $\mathrm{M}$, et al. Análisis comparativo del componente de práctica en el currículo de formación profesional de médicos y profesores en la Pontificia Universidad Católica de Chile. Estudios Pedagógicos 2014; XI (1): 179-96.

3. Prince KJAH, van der Wiel MWJ, Scherpbier AJJA, van der Vleuten CPM, Boshuizen HPA. A qualitative analysis of the transition from theory to practice in undergraduate training in a PBL-medical school. Adv Health Sci Educ 2000; 5: 105-16.

4. Teunissen P, Westerman M. Opportunity or threat: Ambiguity in the consequences of transitions in medical education. Med Educ 2011; 45: 51-9.

5. Helmich E, Bolhuis S, Dornan T, Laan R, Koopmans R Entering medical practice for the very first time: emotional talk, meaning and identity development. Med Educ 2012; 46: 1074-87.

6. McConnell M, Eva K. The role of emotion in the learning and transfer of clinical skills and knowledge. Acad Med 2012; 87 (10): 1316-22.

7. Bitran M, Zúñiga D, Leiva I, Calderón M, Tomicic A, Padilla $\mathrm{O}$, et al. [Perceptions of students and teachers about clinical medicine learning]. Rev Med Chile 2014; 142: 723-31.

8. Prince KJ, Boshuizen HP, Van Der Vleuten CPM, Scherpbiers A. Students' opinions about their preparation for clinical practice. Med Educ 2005; 39 (7): 704-12.

9. Bitran M, Zúñiga D, Lafuente M, Viviani P, Mena B. [Psychological types and learning styles of students entering medical school at the Pontificia Universidad Católica de Chile]. Rev Med Chile 2003; 131: 1067-78.

10. Palacios S, Matus O, Soto A, Ibáñez P, Fasce E. Estilos de aprendizaje en primer año de medicina según cuestionario Honey-Alonso. Publicación preliminar. Rev Educ Ciencias de la Salud 2006; 3: 89-94.

11. Kolb D. Experiential learning: Experience as the source of learning and development. New Jersey: Prentice Hall-PTR, 1984.

12. Kolb D, Boyatzis R, Mainemelis C. Experiential learning theory: Previous research and new directions. En: R. Sternberg \& L. Zhang, Editores, Perspectives on cognitive learning and thinking styles. Mahwah NJ: Erlbaum 2001.p. 228-47.

13. Dornan T, Tan N, Boshuizen H, Gick R, Isba R, Mann $\mathrm{K}$, et al. How and what do medical students learn in clerkships? Experience-based learning (ExBL) Adv Health Sci Educ 2014; 9: 721-49.

14. Kolb AY, Kolb DA. Learning styles and learning spaces: A review of the multidisciplinary application of experiential learning in higher education. Chapter 3. En Sims R and Sims S. Editores, Learning styles and learning: A key to meeting the accountability demands in education. Hauppauge, NY: Nova Publishers 2006; p. 45-91.

15. Fleming S, McKee G, Huntley-Moore S. Undergraduate nursing students' learning styles: a longitudinal study. Nurse Educ Today 2011; 31: 444-9.

16. Bitran $M$, Zúñiga D, Pedrals N, Padilla O, Mena B. Medical students' change in learning styles during the course of undergraduate program: from 'thinking and learning' to 'thinking and doing'. Can Med Educ J 2012; 3 (2): e86-e97.

17. Emilia O, Bloomfield L, Rotem A. Measuring students' approaches to learning in different clinical rotations BMC Medical Education 2012; 12: 114.

18. Hsu C-H, Sandford B. The Delfi technique-making sense of consensus. Practical Assess Res Eval 2007; 12 (10): 1-8.

19. Hasson F, Keeney S, McKenna H. Research guidelines for the Delfi survey technique. J Adv Nurs 2000; 32 (4): 1008-15.

20. Cronbach LJ. Coefficient alpha and the internal structure of tests. Psychometrika 1951; 16: 297-334.

21. Kaiser $H$. The varimax criterion for analytic rotation in factor analysis. Psychometrika 1958; 23: 187-200.

22. Humphreys Lloyd, Montanelli R. An investigation of the parallel analysis criterion for determining the number of common factors. Multivariate Behavioral Research 1975; 10: 193-205.

23. Floyd F, Widaman K. Factor analysis in the development 
and refinement of clinical assessment instruments. Psychological Assessment 1995; 7 (3): 286-99.

24. Field A. Discovering Statistics using SPSS. London: Sage Publications 2006.

25. Yardley S, Teunissen P, Dornan T. AMEE guides: Theories in Medical Education. Experiential Learning AMEE guide No. 63. Med Teach 2012; 34: e102-15.

26. Lave J, Wenger E. Situated learning: Legitimate peripheral participation. Cambridge: Cambridge University Press; 1991.

27. Wenger E. Communities of practice: Learning, meaning and identity. New York, NY: Cambridge University Press; 1998.

28. Ryan R, Deci E. Self-Determination Theory and the Facilitation of Intrinsic Motivation, Social Development, and Well-Being. American Psychologist 2000; 55 (1): 68-78.

29. Dyrbye L, Thomas M, Harper W, Massie F, Power D, Eacker A, et al. The learning environment and medical student burnout: a multicentre study. Med Educ 2009; 43: 274-82.

30. Tanaka M, Fukuda S, Mizuno K, Kuratsune H, Watanabe Y. Stress and coping styles are associated with severe fatigue in medical students. Behavioral Medicine 2009; 35 (3): 87-92.

31. Román C, Rodríguez F, Hernández Y. El estrés académico en estudiantes latinoamericanos de la carrera de medicina. Revista Iberoamericana de Educación 2008; 46 (7): 1-8.

32. Benítez C, Quintero J, Torres R. Prevalencia de riesgo de trastornos psiquiátricos en estudiantes de pregrado de la Escuela de Medicina de la Pontificia Universidad Católica de Chile. Rev Med Chile 2001; 129 (2): 173-8.

33. Romero M, Santander J, Hitschfeld M, Labbé M, Zamora V. Consumo de sustancias ilícitas y psicotrópicos entre los estudiantes de medicina de la Pontificia Universidad Católica de Chile. Rev Med Chile 2009; 137: 459-65.

34. Mavor K, McNeil K, Anderson K, Kerr A, O’Reilly E, Platow M. Beyond prevalence to process: the role of self and identity in medical student well-being. Med Educ 2014; 48: 351-60.

35. Frías-Navarro D, Pascual M. Prácticas del análisis factorial exploratorio (AFE) en la investigación sobre conductas del consumidor y marketing. Suma Psicológica 2012; 19 (1): 47-58.

36. Biggs JB, Kember D, Leung DYP. The revised two-factor Study Process Questionnaire: R-SPQ-2F. Br J Educ Psychol 2001; 71: 133-49.

37. Meyer, J. Study orchestration: the manifestation, interpretation and consequences of contextualised approaches to studying Higher Education1991; 22 (3): 297-316.

38. Everitt, S. Multivariate analysis: The need for data, and other problems. British Journal of Psychiatry, 1975; 126: 237-40.

39. Gorsuch RL. Factor analysis (2nd Ed.). Hillsdale, NJ: Lawrence Erlbaum. 1983.

40. Lloret-Segura S, Ferreres-Traver A, Hernández-Baeza A, Tomas-Marco I. El Análisis Factorial Exploratorio de los Îtems: una guía práctica, revisada y actualizada. Anal. Psicol [online] 2014; 30 (3): 1151-69. 